\title{
THE GEOMETRY OF CONVEX CONES ASSOCIATED WITH THE LYAPUNOV INEQUALITY AND THE COMMON LYAPUNOV FUNCTION PROBLEM*
}

\author{
OLIVER MASON ${ }^{\dagger}$ AND ROBERT SHORTEN ${ }^{\ddagger}$
}

\begin{abstract}
In this paper, the structure of several convex cones that arise in the study of Lyapunov functions is investigated. In particular, the cones associated with quadratic Lyapunov functions for both linear and non-linear systems are considered, as well as cones that arise in connection with diagonal and linear copositive Lyapunov functions for positive linear systems. In each of these cases, some technical results are presented on the structure of individual cones and it is shown how these insights can lead to new results on the problem of common Lyapunov function existence.
\end{abstract}

Key words. Lyapunov functions and stability, Convex cones, Matrix equations.

AMS subject classifications. 37B25, 47L07, 39B42.

1. Introduction and motivation. Recently, there has been considerable interest across the mathematics, computer science, and control engineering communities in the analysis and design of so-called hybrid dynamical systems [7, 15, 19, 22, 23]. Roughly speaking, a hybrid system is one whose behaviour can be described mathematically by combining classical differential/difference equations with some logic based switching mechanism or rule. These systems arise in a wide variety of engineering applications with examples occurring in the aircraft, automotive and communications industries. In spite of the attention that hybrid systems have received in the recent past, important aspects of their behaviour are not yet completely understood. In particular, several questions relating to the stability of hybrid systems have not yet been settled satisfactorily $[7,15]$. Given how pervasive these systems are in practice, in particular in safety critical applications, understanding their stability properties is an issue of paramount importance.

In this paper we consider a number of mathematical problems motivated by the stability of an important and widely studied class of hybrid dynamical systems; namely switched linear systems of the form

$$
\dot{x}(t)=A(t) x(t) \quad A(t) \in \mathcal{A}=\left\{A_{1}, \ldots, A_{m}\right\} \subset \mathbb{R}^{n \times n},
$$

(where $x(t) \in \mathbb{R}^{n}$ ) constructed by switching between a set of linear vector fields. In the stability analysis of both linear and non-linear systems, Lyapunov functions have long played a key role and one approach to establishing the stability of a switched linear system under arbitrary switching rules is to demonstrate that a common Lyapunov

\footnotetext{
* Received by the editors 8 December 2004. Accepted for publication 18 February 2005. Handling Editor: Abraham Berman.

${ }^{\dagger}$ Hamilton Institute, NUI Maynooth, Co. Kildare, Ireland (oliver.mason@nuim.ie). Joint first author.

${ }^{\ddagger}$ Hamilton Institute, NUI Maynooth, Co. Kildare, Ireland (robert.shorten@nuim.ie). Joint first author.
} 
function exists for its constituent linear time-invariant (LTI) systems

$$
\Sigma_{A_{i}}: \dot{x}=A_{i} x, \quad 1 \leq i \leq m .
$$

In this context, it is usual to assume that the constituent LTI systems are all stable, meaning the the eigenvalues of the matrices $A_{i}, 1 \leq i \leq m$ all lie in the open left half plane. Such matrices are said to be Hurwitz.

There are many close links between Lyapunov functions and the theory of convex cones. For instance, a classical result of Lyapunov established that an LTI system $\Sigma_{A}$ is stable if and only if the convex cone

$$
\mathcal{P}_{A}=\left\{P=P^{T}>0: A^{T} P+P A<0\right\}
$$

is non-empty. If $P$ is in $\mathcal{P}_{A}$, then the function $V(x)=x^{T} P x$ is said to be a quadratic Lyapunov function for the system $\Sigma_{A}$. In the context of switched linear systems, the existence of a common quadratic Lyapunov function (CQLF) for the constituent LTI systems $\Sigma_{A_{i}}, 1 \leq i \leq m$ is sufficient for the stability of the system (1.1). Formally, $V(x)=x^{T} P x$ is a CQLF for the LTI systems $\Sigma_{A_{1}}, \ldots, \Sigma_{A_{m}}$ if $P=P^{T}>0$ and

$$
A_{i}^{T} P+P A_{i}<0,
$$

for $1 \leq i \leq m$.

While there are now powerful numerical techniques available [3] that can be used to test whether or not a family of LTI systems has a CQLF, concise analytic conditions for CQLF existence are still quite rare. In fact, this is true even for the case of a pair of LTI systems. Many of the results in the literature on this problem provide conditions for CQLF existence for specific system classes that exploit assumptions about the structure of the system matrices. For instance, results are known for the cases of symmetric, normal or triangular system matrices [21, 28].

The problem of CQLF existence naturally leads to the study of intersections of two or more cones of the form $\mathcal{P}_{A}$. Specifically, given Hurwitz matrices $A_{1}, \ldots, A_{m}$ in $\mathbb{R}^{n \times n}$, the LTI systems $\Sigma_{A_{1}}, \ldots, \Sigma_{A_{m}}$ have a CQLF if and only if the intersection (which is also a convex cone)

$$
\mathcal{P}_{\left\{A_{1}, \ldots, A_{m}\right\}}=\mathcal{P}_{A_{1}} \cap \mathcal{P}_{A_{2}} \cdots \cap \mathcal{P}_{A_{m}}
$$

is non-empty. A number of authors have studied the structure of cones of the form $\mathcal{P}_{\left\{A_{1}, \ldots, A_{m}\right\}}$ and how it relates to the problem of CQLF existence $[5,6,9,14]$. In this context we should also note the work done on the closely related class of cones given by

$$
\mathcal{A}_{P}=\left\{A \in \mathbb{R}^{n \times n}: A^{T} P+P A<0\right\}
$$

for a fixed symmetric matrix $P[1,5]$. Cones of the form $\mathcal{A}_{P}$ belong to the class of so-called convex invertible cones (CICs) and cones of this form have been studied in a recent series of papers wherein many of their basic properties have been elucidated [5, 14]. 
In a recent paper [13], general theoretical conditions for CQLF existence for pairs of LTI systems have been derived. Moreover, the work of this paper indicates how the boundary structure of individual cones of the form $\mathcal{P}_{A}$ can influence the complexity of the conditions for CQLF existence, with the conditions for a general pair of systems being extremely complicated and difficult to check. In this paper, we shall investigate the geometry of cones of the form $\mathcal{P}_{A}$, and present a number of results on the boundary structure of these cones. We shall see how certain properties of the boundary of the cone $\mathcal{P}_{A}$ can give insights into the types of intersections that are possible between two such cones. Moreover we shall see that by making certain simplifying assumptions about the boundary structure of these cones, it is sometimes possible to obtain simple conditions for CQLF existence. We shall also present some preliminary results on convex cones related to the problem of CQLF existence for non-linear systems.

Two other convex cones associated with Lyapunov functions arise in connection with the stability of positive linear systems. The state variables of such systems are constrained to remain non-negative for any non-negative initial conditions, and their stability is known to be equivalent to the existence of both diagonal and linear copositive Lyapunov functions. Both of these types of Lyapunov function are naturally associated with convex cones and we shall present several results on the structure of the relevant cones here as well as indicate the significance of these results for the question of common Lyapunov function existence.

The layout of the paper is as follows. In the next section, we introduce the principal notations used throughout the paper as well as some mathematical background for the work of the paper. In Section 3, we study convex cones associated with quadratic Lyapunov functions for linear systems and indicate the relevance of the structure of such cones to the CQLF existence problem. In Section 4, we turn our attention to the stability of positive linear systems and study convex cones associated with both diagonal Lyapunov functions and linear copositive Lyapunov functions for such systems. In Section 5, we present some initial results on convex cones connected with CQLF existence for non-linear systems. Finally, in Section 6 we present our concluding remarks.

2. Notation and preliminaries. In this section, we introduce some of the main notations used throughout the paper as well as quoting a number of preliminary mathematical results that shall be needed in later sections.

Throughout, $\mathbb{R}$ denotes the field of real numbers, $\mathbb{R}^{n}$ stands for the vector space of all $n$-tuples of real numbers and $\mathbb{R}^{n \times n}$ is the space of $n \times n$ matrices with real entries. Also, $\mathbb{S}^{n \times n}$ and $\mathbb{D}^{n \times n}$ denote the vector spaces of symmetric and diagonal matrices in $\mathbb{R}^{n \times n}$ respectively.

For a vector $x$ in $\mathbb{R}^{n}, x_{i}$ denotes the $i^{\text {th }}$ component of $x$, and the notation $x \succ 0$ $(x \succeq 0)$ means that $x_{i}>0\left(x_{i} \geq 0\right)$ for $1 \leq i \leq n$. Similarly, for a matrix $A$ in $\mathbb{R}^{n \times n}$, $a_{i j}$ denotes the element in the $(i, j)$ position of $A$, and $A \succ 0(A \succeq 0)$ means that $a_{i j}>0\left(a_{i j} \geq 0\right)$ for $1 \leq i, j \leq n$. $A \succ B(A \succeq B)$ means that $A-B \succ 0(A-B \succeq 0)$. The notation $x \prec 0(x \preceq 0)$ means that $-x \succ 0(-x \succeq 0)$.

We shall write $A^{T}$ for the transpose of the matrix $A$ and for $P=P^{T}$ in $\mathbb{R}^{n \times n}$ the notation $P>0$ means that the matrix $P$ is positive definite. 
The spectral radius of a matrix $A$ is denoted by $\rho(A)$ and we shall denote the maximal real part of any eigenvalue of $A$ by $\mu(A)$. Thus $A$ is Hurwitz if and only if $\mu(A)<0$.

\section{Convex cones and tangent hyperplanes:}

A subset $C$ of a real normed vector space $V$ is said to be a convex cone if for all $x, y \in C$ and all real $\lambda>0, \mu>0, \lambda x+\mu y$ is also in $C$. We shall use $\bar{C}$ to denote the closure of $C$ with respect to the norm topology on $V$, and the boundary of $C$ is then defined to be the set difference $\bar{C} / C=\{x \in \bar{C}: x \notin C\}$.

Let $C$ be an open convex cone in $V$. Then for a linear functional $f: V \rightarrow \mathbb{R}$, we say that the corresponding hyperplane through the origin $\mathcal{H}_{f}$ given by

$$
\mathcal{H}_{f}=\{x \in V: f(x)=0\},
$$

is tangential to $C$ at a point $x_{0}$ in its closure $\bar{C}$ if

(i) $f\left(x_{0}\right)=0$;

(ii) $f(x) \neq 0$ for all $x \in C$.

\section{The Lyapunov operator $\mathcal{L}_{A}$ :}

Let $A \in \mathbb{R}^{n \times n}$ be Hurwitz. Then $\mathcal{L}_{A}$ denotes the linear operator defined on the space $\mathbb{S}^{n \times n}$ by

$$
\mathcal{L}_{A}(H)=A^{T} H+H A \quad \text { for all } H \in \mathbb{S}^{n \times n} .
$$

If the eigenvalues of $A \in \mathbb{R}^{n \times n}$ are $\lambda_{1}, \ldots, \lambda_{n}$, then the eigenvalues of $\mathcal{L}_{A}$ are given by $\lambda_{i}+\lambda_{j}$ for $1 \leq i \leq j \leq n$ [11]. In particular, $\mathcal{L}_{A}$ is invertible (in fact all of its eigenvalues lie in the open left half plane) if $A$ is Hurwitz.

\section{Hyperplanes in $\mathbb{S}^{n \times n}$ :}

Finally for this section, we recall the following lemma from [30] which relates two equivalent parameterizations of the same hyperplane in the space $\mathbb{S}^{n \times n}$.

Lemma 2.1. Let $x, y, u, v$ be non-zero vectors in $\mathbb{R}^{n}$. Suppose that there is some $k>0$ such that for all symmetric matrices $P \in \mathbb{S}^{n \times n}$

$$
x^{T} P y=-k u^{T} P v .
$$

Then either

$$
x=\alpha u \text { for some real scalar } \alpha, \text { and } y=-\left(\frac{k}{\alpha}\right) v
$$

or

$$
x=\beta v \text { for some real scalar } \beta \text { and } y=-\left(\frac{k}{\beta}\right) u \text {. }
$$


3. CQLF existence and the cones $\mathcal{P}_{A}$. In this section, we study the cone

$$
\mathcal{P}_{A}=\left\{P=P^{T}>0: A^{T} P+P A<0\right\}
$$

for a Hurwitz matrix $A$ in $\mathbb{R}^{n \times n}$. In particular, we present some initial results on the boundary structure of the cone $\mathcal{P}_{A}$ and indicate the relevance of these results to the CQLF existence problem for pairs of stable LTI systems. We also present a number of related facts about possible intersections between two cones $\mathcal{P}_{A_{1}}, \mathcal{P}_{A_{2}}$ where $A_{1}$ and $A_{2}$ are both Hurwitz as well as a technical fact about the left and right eigenvectors of singular matrix pencils that follows from results on CQLF existence.

Note that the closure of the open convex cone $\mathcal{P}_{A}$ (with respect to the topology on $\mathbb{S}^{n \times n}$ given by the matrix norm induced from the usual Euclidean norm on $\mathbb{R}^{n}$ ) is given by

$$
\left\{P=P^{T} \geq 0: A^{T} P+P A \leq 0\right\},
$$

and the boundary of $\mathcal{P}_{A}$ is

$$
\left\{P=P^{T} \geq 0: A^{T} P+P A \leq 0, \operatorname{det}\left(A^{T} P+P A\right)=0\right\} .
$$

The cones $\mathcal{P}_{A}$ and $\mathcal{P}_{A^{-1}}$ :

The following result, derived by Loewy in [16], completely characterises pairs of Hurwitz matrices $A, B$ in $\mathbb{R}^{n \times n}$ for which $\mathcal{P}_{A}=\mathcal{P}_{B}$.

Theorem 3.1. Let $A, B$ be Hurwitz matrices in $\mathbb{R}^{n \times n}$. Then $\mathcal{P}_{B}=\mathcal{P}_{A}$ if and only if $B=\mu A$ for some real $\mu>0$ or $B=\lambda A^{-1}$ for some real $\lambda>0$.

An immediate consequence of Theorem 3.1 is that, for a Hurwitz matrix $A$, the two sets $\mathcal{P}_{A}$ and $\mathcal{P}_{A^{-1}}$ are identical. This means that a result on CQLF existence for a family of LTI systems $\Sigma_{A_{1}}, \ldots, \Sigma_{A_{k}}$ can also be applied to any family of systems obtained by replacing some of the matrices $A_{i}$ with their inverses $A_{i}^{-1}$.

\section{Tangent hyperplanes to $\mathcal{P}_{A}$ :}

We shall now consider hyperplanes in $\mathbb{S}^{n \times n}$ that are tangential to the cone $\mathcal{P}_{A}$. Specifically, we shall characterize those hyperplanes that are tangential to the cone $\mathcal{P}_{A}$ at certain points in its boundary, and show how an interesting result on CQLF existence follows in a natural way from this characterization.

Now, consider a point $P_{0}$ in the closure of $\mathcal{P}_{A}$ for which $A^{T} P_{0}+P_{0} A \leq 0$ has rank $n-1$. The next result shows that, in this case, there is a unique hyperplane tangential to $\mathcal{P}_{A}$ that passes through $P_{0}$, and moreover that this hyperplane can be parameterized in a natural way.

Theorem 3.2. Let $A \in \mathbb{R}^{n \times n}$ be Hurwitz. Suppose that $A^{T} P_{0}+P_{0} A=Q_{0} \leq 0$ and $\operatorname{rank}\left(Q_{0}\right)=n-1$, with $\left(A^{T} P_{0}+P_{0} A\right) x_{0}=0, x_{0} \neq 0$. Then:

(i) there is a unique hyperplane tangential to $\mathcal{P}_{A}$ at $P_{0}$;

(ii) this hyperplane is given by

$$
\left\{H \in \mathbb{S}^{n \times n}: x_{0}^{T} H A x_{0}=0\right\} .
$$


Proof. Let

$$
\mathcal{H}_{f}=\left\{H \in \mathbb{S}^{n \times n}: f(H)=0\right\}
$$

be a hyperplane that is tangential to $\mathcal{P}_{A}$ at $P_{0}$, where $f$ is a linear functional defined on $\mathbb{S}^{n \times n}$. We shall show that $\mathcal{H}_{f}$ must coincide with the hyperplane

$$
\mathcal{H}=\left\{H \in \mathbb{S}^{n \times n}: x_{0}^{T} H A x_{0}=0\right\} .
$$

Suppose that this was not true. This would mean that there was some $\bar{P}$ in $\mathbb{S}^{n \times n}$ such that $f(\bar{P})=0$ but $x_{0}^{T} \bar{P} A x_{0}<0$.

Now, consider the set

$$
\Omega=\left\{x \in \mathbb{R}^{n}: x^{T} x=1 \text { and } x^{T} \bar{P} A x \geq 0\right\},
$$

and note that if $\Omega$ was empty, this would mean that $\bar{P}$ was in $\mathcal{P}_{A}$, contradicting the fact that $\mathcal{H}_{f}$ is tangential to $\mathcal{P}_{A}$. Thus, we can assume that $\Omega$ is non-empty.

Note that the set $\Omega$ is closed and bounded, hence compact. Furthermore $x_{0}$ is not in $\Omega$ and thus $x^{T} P_{0} A x<0$ for all $x$ in $\Omega$.

Let $M_{1}$ be the maximum value of $x^{T} \bar{P} A x$ on $\Omega$, and let $M_{2}$ be the maximum value of $x^{T} P_{0} A x$ on $\Omega$. Then by the final remark in the previous paragraph, $M_{2}<0$. Choose any constant $\delta>0$ such that

$$
\delta<\frac{\left|M_{2}\right|}{M_{1}+1}=C_{1}
$$

and consider the symmetric matrix

$$
P_{0}+\delta_{1} \bar{P}
$$

By separately considering the cases $x \in \Omega$ and $x \notin \Omega, x^{T} x=1$, it follows that for all non-zero vectors $x$ of Euclidean norm 1

$$
x^{T}\left(A^{T}\left(P_{0}+\delta \bar{P}\right)+\left(P_{0}+\delta \bar{P}\right) A\right) x<0
$$

provided $0<\delta<\frac{\left|M_{2}\right|}{M_{1}+1}$. Since the above inequality is unchanged if we scale $x$ by any non-zero real number, it follows that $A^{T}\left(P_{0}+\delta \bar{P}\right)+\left(P_{0}+\delta \bar{P}\right) A$ is negative definite.

Thus, $P_{0}+\delta \bar{P}$ is in $\mathcal{P}_{A}$. However,

$$
f\left(P_{0}+\delta \bar{P}\right)=f\left(P_{0}\right)+\delta f(\bar{P})=0
$$

which implies that $\mathcal{H}_{f}$ intersects the interior of the cone $\mathcal{P}_{A}$ which is a contradiction. Thus, there can be only one hyperplane tangential to $\mathcal{P}_{A}$ at $P_{0}$, and this is given by

$$
\left\{H \in \mathbb{S}^{n \times n}: x_{0}^{T} H A x_{0}=0\right\},
$$

as claimed. 


\section{Tangent hyperplanes and the CQLF existence problem:}

We now show the relevance of the previous result on the structure of the cone $\mathcal{P}_{A}$ to the problem of CQLF existence. Specifically, we demonstrate how it leads in a natural way to a result that has previously appeared in [30].

Let $A_{1}, A_{2} \in \mathbb{R}^{n \times n}$ be two Hurwitz matrices such that the LTI systems $\Sigma_{A_{1}}, \Sigma_{A_{2}}$ do not have a CQLF. Further assume that there does exist a positive semi-definite $P=P^{T} \geq 0$ such that $A_{i}^{T} P+P A_{i}=Q_{i} \leq 0$, with $\operatorname{rank}\left(Q_{i}\right)=n-1$ for $i=1,2$. Then:

(i) there exists a hyperplane, $\mathcal{H}$, through the origin in $\mathbb{S}^{n \times n}$ that separates the disjoint open convex cones $\mathcal{P}_{A_{1}}, \mathcal{P}_{A_{2}}[25]$

(ii) any hyperplane separating $\mathcal{P}_{A_{1}}, \mathcal{P}_{A_{2}}$ must contain the matrix $P$, and be tangential to both $\mathcal{P}_{A_{1}}$ and $\mathcal{P}_{A_{2}}$ at $P$

(iii) there exist non-zero vectors $x_{1}, x_{2}$ in $\mathbb{R}^{n}$ such that $Q_{i} x_{i}=0$ for $i=1,2$.

Now on combining (i) and (ii) with Theorem 3.2, we can see that in fact there is a unique hyperplane $\mathcal{H}$ separating $\mathcal{P}_{A_{1}}, \mathcal{P}_{A_{2}}$. Furthermore, we can use (iii) and Theorem 3.2 to parameterize $\mathcal{H}$ in two different ways. Namely:

$$
\begin{aligned}
\mathcal{H} & =\left\{H \in \mathbb{S}^{n \times n}: x_{1}^{T} H A_{1} x_{1}=0\right\} \\
& =\left\{H \in \mathbb{S}^{n \times n}: x_{2}^{T} H A_{2} x_{2}=0\right\} .
\end{aligned}
$$

It now follows that there must be some constant $k>0$ such that

$$
x_{1}^{T} H A_{1} x_{1}=-k x_{2}^{T} H A_{2} x_{2},
$$

for all $H$ in $\mathbb{S}^{n \times n}$. Applying Lemma 2.1 now immediately yields the following result.

TheOREM 3.3. [30] Let $A_{1}, A_{2}$ be Hurwitz matrices in $\mathbb{R}^{n \times n}$ such that $\Sigma_{A_{1}}, \Sigma_{A_{2}}$ do not have a CQLF. Furthermore, suppose that there is some $P=P^{T} \geq 0$ such that

$$
A_{i}^{T} P+P A_{i}=Q_{i} \leq 0, i \in\{1,2\}
$$

for some negative semi-definite matrices $Q_{1}, Q_{2}$ in $\mathbb{R}^{n \times n}$, both of rank $n-1$. Under these conditions, at least one of the matrix products $A_{1} A_{2}$ and $A_{1} A_{2}^{-1}$ has a negative real eigenvalue.

\section{Boundary structure of $\mathcal{P}_{A}$ :}

In the following lemma, we examine the assumption that the rank of $A_{i}^{T} P+P A_{i}$ is $n-1$ for $i=1,2$ in Theorem 3.3. In particular, we note that those matrices $P$ such that $A^{T} P+P A=Q \leq 0$ with $\operatorname{rank}(Q)=n-1$ are dense in the boundary of $\mathcal{P}_{A}$. So, in a sense, the 'rank $n-1$ ' assumption of Theorem 3.3 is not overly restrictive. In the statement of the lemma, $\|\cdot\|$ denotes the matrix norm on $\mathbb{R}^{n \times n}$ induced by the usual Euclidean norm on $\mathbb{R}^{n}[10]$.

Lemma 3.4. Let $A \in \mathbb{R}^{n \times n}$ be Hurwitz, and suppose that $P=P^{T} \geq 0$ is such that $A^{T} P+P A \leq 0$ and $\operatorname{rank}\left(A^{T} P+P A\right)=n-k$ for some $k$ with $1<k \leq n$. Then for any $\epsilon>0$, there exists some $P_{0}=P_{0}^{T} \geq 0$ such that: 
(i) $\left\|P-P_{0}\right\|<\epsilon$;

(ii) $A^{T} P_{0}+P_{0} A=Q_{0} \leq 0$;

(iii) $\operatorname{rank}\left(Q_{0}\right)=n-1$.

Proof. Let $Q=A^{T} P+P A$, and note that as the inverse, $\mathcal{L}_{A}^{-1}$, of the Lyapunov operator $\mathcal{L}_{A}$ is continuous, there is some $\delta>0$ such that if $\left\|Q^{\prime}-Q\right\|<\delta$, then $\left\|\mathcal{L}_{A}^{-1}\left(Q^{\prime}\right)-\mathcal{L}_{A}^{-1}(Q)\right\|<\epsilon$. Now, as $Q$ is symmetric and has rank $n-k$, there exists some orthogonal matrix $T$ in $\mathbb{R}^{n \times n}$ such that

$$
\tilde{Q}=T^{T} Q T=\operatorname{diag}\left\{\lambda_{1}, \ldots, \lambda_{n-k}, 0, \ldots, 0\right\},
$$

where $\lambda_{1}<0, \ldots, \lambda_{n-k}<0$. Now, define

$$
\tilde{Q}_{0}=\operatorname{diag}\left\{\lambda_{1}, \ldots, \lambda_{n-k},-\delta / 2, \ldots,-\delta / 2,0\right\},
$$

and let $Q_{0}=T \tilde{Q}_{0} T^{T}$. Then we have that:

(i) $Q_{0} \leq 0$, and $\operatorname{rank}\left(Q_{0}\right)=n-1$;

(ii) $\left\|Q-Q_{0}\right\|<\delta$.

It now follows that the matrix $P_{0}=\mathcal{L}_{A}^{-1}\left(Q_{0}\right)$ lies on the boundary of $\mathcal{P}_{A}$ and satisfies:

(i) $\left\|P_{0}-P\right\|<\epsilon$;

(ii) $\operatorname{rank}\left(A^{T} P_{0}+P_{0} A\right)=n-1$, as required.

\section{Necessary and sufficient conditions for CQLF existence:}

Two of the most significant classes of systems for which simple verifiable conditions for CQLF existence are known are the classes of second order systems [4, 31] and systems whose system matrices are in companion form [24, 29]. In a number of recent papers [27,30], it has been demonstrated that, in a sense, Theorem 3.3 provides a unifying framework for both of these results.

Second order systems:

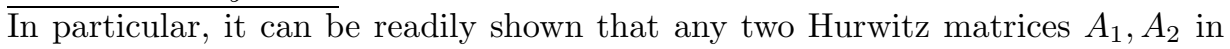
$\mathbb{R}^{2 \times 2}$ such that:

(i) the LTI systems $\Sigma_{A_{1}}, \Sigma_{A_{2}}$ do not have a CQLF;

(ii) for any $\alpha>0$, the LTI systems $\Sigma_{A_{1}}, \Sigma_{A_{2}-\alpha I}$ have a CQLF, will satisfy the conditions of Theorem 3.3. This fact can be used to give a simple proof of the known result $[4,31]$ that two stable second order LTI systems $\Sigma_{A_{1}}, \Sigma_{A_{2}}$ $\left(A_{1}, A_{2} \in \mathbb{R}^{2 \times 2}\right)$ have a CQLF if and only if the matrix products $A_{1} A_{2}$ and $A_{1} A_{2}^{-1}$ have no negative real eigenvalues.

Systems differing by rank one:

Moreover, it has been shown in [27] that Theorem 3.3 can also be applied generically ${ }^{1}$ to the case of a pair of Hurwitz matrices $A, A-g h^{T} \in \mathbb{R}^{n \times n}$ in companion form. More specifically, let $A, A-g h^{T}$ be two such matrices in $\mathbb{R}^{n \times n}$ such that:

(i) the LTI systems $\Sigma_{A}, \Sigma_{A-g h^{T}}$ do not have a CQLF;

(ii) for any $k$ with $0<k<1$, the LTI systems $\Sigma_{A}, \Sigma_{A-k g h^{T}}$ have a CQLF.

\footnotetext{
${ }^{1}$ Essentially, we need to assume that the entries of the system matrices do not satisfy a specific polynomial equation. For details consult [27].
} 
Then for all $\epsilon>0$, there exists some $h^{\prime} \in \mathbb{R}^{n}$ with $\left\|h-h^{\prime}\right\|<\epsilon$ such that $A$ and $A-g h^{\prime T}$ satisfy the hypotheses of Theorem 3.3. This fact can then be used to show that a necessary and sufficient condition for a pair of companion matrices $A, A-g h^{T}$ in $\mathbb{R}^{n \times n}$ to have a CQLF is that the matrix product $A\left(A-g h^{T}\right)$ has no negative real eigenvalues. In fact, the following result on CQLF existence for a pair of stable LTI systems (not necessarily in companion form) differing by a rank one matrix has been derived in [27].

TheOREM 3.5. Let $A_{1}, A_{2}$ be Hurwitz matrices in $\mathbb{R}^{n \times n}$ with $\operatorname{rank}\left(A_{2}-A_{1}\right)=1$. Then the LTI systems $\Sigma_{A_{1}}, \Sigma_{A_{2}}$ have a CQLF if and only if the matrix product $A_{1} A_{2}$ has no negative real eigenvalues.

\section{Simultaneous solutions to Lyapunov equations:}

In Theorem 3.3, we considered a pair of Hurwitz matrices $A_{1}, A_{2}$ in $\mathbb{R}^{n \times n}$ for which there exists some $P=P^{T} \geq 0$ such that $A_{i}^{T} P+P A_{i}=Q_{i} \leq 0$ with $\operatorname{rank}\left(Q_{i}\right)=$ $n-1$ for $i=1,2$. In the following lemma we again investigate the question of simultaneous solutions to a pair of Lyapunov equations. Specifically, we consider a pair of Hurwitz matrices $A_{1}, A_{2}$ in $\mathbb{R}^{n \times n}$ with $\operatorname{rank}\left(A_{2}-A_{1}\right)=1$ and demonstrate that in this situation, there can exist no $P=P^{T}>0$ that simultaneously satisfies

$$
\begin{aligned}
& A_{1}^{T} P+P A_{1}=Q_{1}<0 \\
& A_{2}^{T} P+P A_{2}=Q_{2} \leq 0
\end{aligned}
$$

with $\operatorname{rank}\left(Q_{2}\right)<n-1$.

Lemma 3.6. Let $A_{1} \in \mathbb{R}^{n \times n}$ be Hurwitz and suppose that $P$ is in the boundary of $\mathcal{P}_{A_{1}}$ with

$$
A_{1}^{T} P+P A_{1}=Q_{1} \leq 0
$$

Then if $P \in \mathcal{P}_{A_{2}}$ for some Hurwitz matrix $A_{2} \in \mathbb{R}^{n \times n}$ with $\operatorname{rank}\left(A_{2}-A_{1}\right)=1$, the rank of $Q_{1}$ must be $n-1$.

Proof. Let $B=A_{2}-A_{1}$. To begin with, we assume that $B$ is in Jordan canonical form so that (as $B$ is of rank 1) either

$$
B=\left(\begin{array}{cccc}
\lambda & 0 & \ldots & 0 \\
0 & \ldots & \ldots & 0 \\
\vdots & & & \\
0 & \ldots & \ldots & 0
\end{array}\right)
$$

for some $\lambda \in \mathbb{R}$, or

$$
B=\left(\begin{array}{cccc}
0 & \ldots & \ldots & 0 \\
1 & \ldots & \ldots & 0 \\
\vdots & & & \\
0 & \ldots & \ldots & 0
\end{array}\right)
$$


Now partition $Q_{1}=A_{1}^{T} P+P A_{1}$ as

$$
Q_{1}=\left(\begin{array}{cc}
c_{1} & q_{1}^{T} \\
q_{1} & Q
\end{array}\right)
$$

where $c_{1} \in \mathbb{R}, q_{1} \in \mathbb{R}^{n-1}$ and $Q$ is a symmetric matrix in $\mathbb{R}^{(n-1) \times(n-1)}$. It can be verified by direct computation that $Q_{2}=A_{2}^{T} P+P A_{2}$ takes the form

$$
Q_{2}=\left(\begin{array}{cc}
c_{2} & q_{2}^{T} \\
q_{2} & Q
\end{array}\right)
$$

with the same $Q$ as before.

From the interlacing theorem for bordered symmetric matrices [10], it follows that the eigenvalues of $Q_{i}$ for $i=1,2$ must interlace with the eigenvalues of $Q$. However as $P \in \mathcal{P}_{A_{2}}, Q_{2}<0$ and thus $Q$ must be non-singular in $\mathbb{R}^{(n-1) \times(n-1)}$. Therefore, as the eigenvalues of $Q_{1}$ must also interlace with the eigenvalues of $Q$, it follows that $Q_{1}$ cannot have rank less than $n-1$.

Now suppose that $B$ is not in Jordan canonical form and write $\Lambda=T^{-1} B T$ where $\Lambda$ is in one of the forms (3.6), (3.7). Consider $\tilde{A}_{1}, \tilde{A}_{2}$ and $\tilde{P}$ given by

$$
\tilde{A_{1}}=T^{-1} A_{1} T, \tilde{A_{2}}=T^{-1} A_{2} T, \tilde{P}=T^{T} P T .
$$

Then it is a straightforward exercise in congruences to verify that

$$
{\tilde{A_{2}}}^{T} \tilde{P}+\tilde{P} \tilde{A}_{2}=T^{T} Q_{2} T<0
$$

and that

$$
\tilde{A}_{1}^{T} \tilde{P}+\tilde{P} \tilde{A}_{1}=T^{T} Q_{1} T \leq 0 .
$$

Furthermore $\operatorname{rank}\left(\tilde{A}_{1}-\tilde{A}_{2}\right)=1$ and $\tilde{A}_{1}, \tilde{A}_{2}$ are both Hurwitz. Hence by the previous argument, $T^{T} Q_{1} T$ must have rank $n-1$, and thus by congruence the rank of $Q_{1}$ must also be $n-1$.

REMARK 3.7. The above result shows that for Hurwitz matrices $A_{1}, A_{2} \in \mathbb{R}^{n \times n}$ with $\operatorname{rank}\left(A_{2}-A_{1}\right)=1$, there are definite restrictions on the type of simultaneous solutions possible to the two corresponding Lyapunov inequalities. In fact, it can be seen from examining the proof of the lemma that there can be no solution $P=P^{T} \geq 0$ with $A_{1}^{T} P+P A_{1}=Q_{1} \leq 0, A_{2}^{T} P+P A_{2}=Q_{2} \leq 0$ and $\left|\operatorname{rank}\left(Q_{2}\right)-\operatorname{rank}\left(Q_{1}\right)\right| \geq 2$.

\section{Right and left eigenvectors of singular matrix pencils:}

Finally for this section, we note a curious technical fact about the left and right eigenvectors of singular matrix pencils, which follows from Theorem 3.5.

TheOREM 3.8. Let $A_{1}, A_{2}$ be Hurwitz matrices in $\mathbb{R}^{n \times n}$ with $\operatorname{rank}\left(A_{2}-A_{1}\right)=1$. Suppose that there is exactly one value of $\gamma_{0}>0$ for which $A_{1}^{-1}+\gamma_{0} A_{2}$ is singular. Then for this $\gamma_{0}$ : 
(i) Up to scalar multiples, there exist unique vectors $x_{0} \in \mathbb{R}^{n}, y_{0} \in \mathbb{R}^{n}$ such that

$$
\left(A_{1}^{-1}+\gamma_{0} A_{2}\right) x_{0}=0, \quad y_{0}^{T}\left(A_{1}^{-1}+\gamma_{0} A_{2}\right)=0
$$

(the left and right eigenspaces are one dimensional)

(ii) for this $x_{0}$ and $y_{0}$, it follows that

$$
y_{0}^{T} A_{1}^{-1} x_{0}=0, \quad y_{0}^{T} A_{2} x_{0}=0 .
$$

Proof. Write $B=A_{2}-A_{1}$. Without loss of generality, we can take $B$ to be in Jordan canonical form. Note that the hypotheses of the theorem mean that $\operatorname{det}\left(A_{1}^{-1}+\right.$ $\left.\gamma A_{2}\right)=\operatorname{det}\left(A_{1}^{-1}+\gamma A_{1}+\gamma B\right)$ never changes sign for $\gamma>0$, as $A_{1}^{-1}$ and $A_{2}$ are both Hurwitz. We assume that $\operatorname{det}\left(A_{1}^{-1}+\gamma A_{1}+\gamma B\right) \geq 0$ for all $\gamma>0$. (The case $\operatorname{det}\left(A_{1}^{-1}+\gamma A_{1}+\gamma B\right) \leq 0$ for all $\gamma>0$ may be proven identically.)

Now, for $k>0, \gamma \geq 0$, we can write

$$
\operatorname{det}\left(\left(A_{1}^{-1}+\gamma\left(A_{1}+k B\right)\right)=M(\gamma)+k N(\gamma)\right.
$$

where $M$ and $N$ are polynomials in $\gamma$ with:

(i) $M(\gamma)=\operatorname{det}\left(A_{1}^{-1}+\gamma A_{1}\right)>0$ for all $\gamma>0\left(\left(A_{1}^{-1}+\gamma A_{1}\right)\right.$ is always Hurwitz for $\gamma>0)$;

(ii) $M(0)+k N(0)=M(0)>0$ for any $k>0>0$.

Now, if for some $k$ with $0<k<1$, $\operatorname{det}\left(A_{1}^{-1}+\gamma\left(A_{1}+k B\right)\right)=0$ for some $\gamma>0$, then it follows from (i), (ii) and (3.8) that for the same $\gamma, \operatorname{det}\left(A_{1}^{-1}+\gamma_{0}\left(A_{1}+B\right)\right)<0$ which contradicts the hypotheses of the Theorem. Thus, for all $k$, with $0<k<1$,

$$
\operatorname{det}\left(A_{1}^{-1}+\gamma\left(A_{1}+k B\right)\right)>0
$$

for all $\gamma>0$. But by Theorem 3.5, this means that for $0<k<1$ there exists a CQLF for the LTI systems $\Sigma_{A_{1}}$ and $\Sigma_{A_{2}}$ and hence, by Theorem 3.1, for the systems $\Sigma_{A_{1}^{-1}}$ and $\Sigma_{A_{2}}$.

It now follows from the results of Meyer, [20] that there must exist some $P=$ $P^{T}>0$ such that

$$
\begin{array}{r}
A_{1}^{-T} P+P A_{1}^{-1} \leq 0 \\
A_{2}^{T} P+P A_{2} \leq 0 .
\end{array}
$$

Furthermore as $x_{0}^{T} P\left(A_{1}+\gamma_{0} A_{2}\right) x_{0}=0$, it follows that

$$
\left(A_{1}^{-T}+\gamma_{0} A_{2}^{T}\right) P x_{0}+P\left(A_{1}^{-1}+\gamma_{0} A_{2}\right) x_{0}=0 .
$$

But, $\left(A_{1}^{-1}+\gamma_{0} A_{2}\right) x_{0}=0$ and hence we must have,

$$
\left(A_{1}^{-T}+\gamma_{0} A_{2}^{T}\right) P x_{0}=0,
$$

and $P x_{0}=\lambda y_{0}$ for some real $\lambda \neq 0$. Now,

$$
y_{0}^{T} A_{1}^{-1} x_{0}+\gamma_{0} y_{0}^{T} A_{2} x_{0}=0
$$


implies that

$$
x_{0}^{T} P A_{1}^{-1} x_{0}+\gamma_{0} x_{0}^{T} P A_{2} x_{0}=0,
$$

from which we can conclude the result of the theorem. $\square$

REMARK 3.9. The above result shows that the left and right eigenvectors $x_{0}$ and $y_{0}$ of the singular pencil at $\gamma_{0}$ are quite strongly constrained.

4. Positive switched systems, diagonal and copositive Lyapunov functions. A dynamical system is said to be positive if its state vector is constrained to remain within the non-negative orthant for all non-negative initial conditions. This class of systems is of considerable importance and arises in numerous applications, including communications, economics, biology and ecology $[8,12,17,26]$. In this section, we turn our attention to problems motivated by the stability of the class of positive switched linear systems constructed by switching between a family of positive LTI systems.

Two special types of Lyapunov functions arise in connection with the study of positive linear systems. Specifically, it is natural to consider diagonal Lyapunov functions and copositive Lyapunov functions when analysing the stability of such systems. We shall see below how such Lyapunov functions are related to certain convex cones and how to exploit this relationship to derive results on common diagonal Lyapunov function (CDLF) and common copositive Lyapunov function existence for pairs of positive LTI systems. First of all, we recall some basic facts about positive LTI systems and their stability.

\section{Positive LTI systems and Metzler matrices:}

An LTI system $\Sigma_{A}$ is positive if and only if the system matrix $A$ is a so-called Metzler matrix [8], meaning that $a_{i j} \geq 0$ for $i \neq j$. The next result recalls some fundamental facts about Metzler matrices, positive LTI systems and stability. In the statement of the following theorem, $\mathbb{D}^{n \times n}$ denotes the space of diagonal matrices in $\mathbb{R}^{n \times n}$.

Theorem 4.1. [2, 8] Let $A \in \mathbb{R}^{n \times n}$ be Metzler. Then the following statements are equivalent.

(i) $A$ is Hurwitz.

(ii) There is some vector $v \succ 0$ in $\mathbb{R}^{n}$ such that $A^{T} v \prec 0$.

(iii) $-A^{-1}$ is a non-negative matrix.

(iv) There is some positive definite diagonal matrix $D$ in $\mathbb{D}^{n \times n}$ such that $A^{T} D+$ $D A<0$.

Thus, three convex cones arise in connection with the stability of positive linear systems; namely the cone $\mathcal{P}_{A}$ studied in the previous section, the cone of diagonal solutions to the Lyapunov inequality and the cone of vectors $v \succ 0$ with $A^{T} v \prec 0$. In the remainder of this section, we shall study the second and third of these cones and indicate how their structure can provide insights into the problem of common Lyapunov function existence for positive systems. 


\section{Irreducible Metzler matrices:}

Later in this section, we shall derive a necessary and sufficient condition for common diagonal Lyapunov function (CDLF) existence for a pair of positive LTI systems whose system matrices are irreducible. Recall that a matrix $A \in \mathbb{R}^{n \times n}$ is said to be reducible [10] if there exists a permutation matrix $P \in \mathbb{R}^{n \times n}$ and some $r$ with $1 \leq r<n$ such that $P A P^{T}$ has the form

$$
\left(\begin{array}{cc}
A_{11} & A_{12} \\
0 & A_{22}
\end{array}\right)
$$

where $A_{11} \in \mathbb{R}^{r \times r}, A_{22} \in \mathbb{R}^{(n-r) \times(n-r)}, A_{12} \in \mathbb{R}^{r \times(n-r)}$ and 0 is the zero matrix in $\mathbb{R}^{(n-r) \times r}$. If a matrix is not reducible, then it is said to be irreducible. We shall later make use of the following fundamental result for irreducible Metzler matrices which corresponds to the Perron Frobenius Theorem for irreducible non-negative matrices [10].

TheOREM 4.2. Let $A \in \mathbb{R}^{n \times n}$ be Metzler and irreducible. Then

(i) $\mu(A)$ is an eigenvalue of $A$ of algebraic (and geometric) multiplicity one;

(ii) there is an eigenvector $x \succ 0$ with $A x=\mu(A) x$.

4.1. The convex cones $\mathcal{D}_{A}$ and common diagonal Lyapunov functions. Theorem 4.1 establishes that a positive LTI system $\Sigma_{A}$ is stable if and only if the convex cone in $\mathbb{D}^{n \times n}$, given by

$$
\mathcal{D}_{A}=\left\{D \in \mathbb{D}^{n \times n}: D>0, A^{T} D+D A<0\right\}
$$

is non-empty. In view of this fact, when studying the stability of positive switched linear systems, it is natural to consider the problem of common diagonal Lyapunov function (CDLF) existence. Formally, given Metzler, Hurwitz matrices $A_{1}, \ldots, A_{m}$ in $\mathbb{R}^{n \times n}$, determine necessary and sufficient conditions for the existence of a single positive definite matrix $D \in \mathbb{D}^{n \times n}$ such that $A_{i}^{T} D+D A_{i}<0$ for $1 \leq i \leq m$. We shall concentrate on the problem of CDLF existence for a pair of stable positive LTI systems $(m=2)$.

\section{Tangent hyperplanes to the cone $\mathcal{D}_{A}$ :}

In the next result, we consider hyperplanes in $\mathbb{D}^{n \times n}$ that are tangential to the cone $\mathcal{D}_{A}$ at points $D$ on its boundary for which $A^{T} D+D A$ has rank $n-1$. As in Theorem 3.2, there is a unique such hyperplane in this case and, moreover, this plane can be parameterized in a natural way. We omit the proof of this result as it is practically identical to the proof of Theorem 3.2 given above.

TheOREM 4.3. Let $A \in \mathbb{R}^{n \times n}$ be Metzler and Hurwitz. Suppose that $D_{0}$ lies on the boundary of the cone $\mathcal{D}_{A}$, and that the rank of $A^{T} D_{0}+D_{0} A$ is $n-1$, with $\left(A^{T} D_{0}+D_{0} A\right) x_{0}=0, x_{0} \neq 0$. Then:

(i) there is a unique hyperplane tangential to $\mathcal{D}_{A}$ at $D_{0}$; 
(ii) this plane is given by

$$
\left\{D \in \mathbb{D}^{n \times n}: x_{0}^{T} D A x_{0}=0\right\} .
$$

\section{The boundary structure of $\mathcal{D}_{A}$ :}

We have seen in Lemma 3.4 that for a Hurwitz matrix $A$ in $\mathbb{R}^{n \times n}$, those matrices $P$ on the boundary of $\mathcal{P}_{A}$ for which the rank of $A^{T} P+P A$ is $n-1$ are dense in the boundary. For the case of an irreducible Metzler Hurwitz matrix $A$ and the cone $\mathcal{D}_{A}$, we can say even more than this.

We shall show below that for an irreducible Metzler, Hurwitz matrix $A$ in $\mathbb{R}^{n \times n}$, and any non-zero diagonal matrix $D$ in the boundary of $\mathcal{D}_{A}$, the rank of $A^{T} D+D A$ must be $n-1$. The first step is the following simple observation.

Lemma 4.4. Let $A \in \mathbb{R}^{n \times n}$ be a Metzler matrix. Then for any diagonal matrix $D$ in $\mathbb{R}^{n \times n}$ with non-negative entries, $A^{T} D+D A$ is also Metzler.

The next result is concerned with diagonal matrices $D$ on the boundary of the set $\mathcal{D}_{A}$, for irreducible Metzler Hurwitz matrices $A$. It establishes that, for such $A$, any non-zero diagonal $D \geq 0$ such that $A^{T} D+D A \leq 0$ must in fact be positive definite.

Lemma 4.5. Let $A$ in $\mathbb{R}^{n \times n}$ be Metzler, Hurwitz and irreducible. Suppose that $A^{T} D+D A \leq 0$ for some non-zero diagonal $D$ in $\mathbb{R}^{n \times n}$. Then $D>0$.

Proof. The key fact in the proof of this result is that if $Q \in \mathbb{R}^{n \times n}$ is positive semi-definite, and for some $i=1, \ldots, n, q_{i i}=0$, then $q_{i j}=0$ for $1 \leq j \leq n[10]$.

We argue by contradiction. Suppose that $D$ is not positive definite. Then we may select a permutation matrix $P$ in $\mathbb{R}^{n \times n}$ such that

$$
D^{\prime}=P D P^{T}=\operatorname{diag}\left\{d_{1}^{\prime}, \ldots, d_{n}^{\prime}\right\}
$$

with $d_{1}^{\prime}=0, \ldots, d_{r}^{\prime}=0$ and $d_{r+1}^{\prime}>0, \ldots, d_{n}^{\prime}>0$, for some $r$ with $1 \leq r<n$. It follows by congruence that writing $A^{\prime}=P A P^{T}$, we have

$$
A^{\prime T} D^{\prime}+D^{\prime} A^{\prime} \leq 0
$$

The $(i, j)$ entry of $A^{\prime T} D^{\prime}+D^{\prime} A^{\prime}$ is given by $a_{i j}^{\prime} d_{i}^{\prime}+d_{j}^{\prime} a_{j i}^{\prime}$. Now for $i=1, \ldots, r, d_{i}^{\prime}=0$ and hence the corresponding diagonal entry, $2 d_{i}^{\prime} a_{i i}^{\prime}$, of $A^{\prime T} D^{\prime}+D^{\prime} A^{\prime}$ is zero. From the remarks at the start of the proof, it now follows that for $1 \leq j \leq n, a_{i j}^{\prime} d_{i}^{\prime}+d_{j}^{\prime} a_{j i}^{\prime}=0$ also, and in particular that for $j=r+1, \ldots, n, a_{j i}^{\prime}=0$.

To summarize, we have shown that if $D$ is not positive definite, then there is some permutation matrix $P$, and some $r$ with $1 \leq r<n$ such that for $i=1, \ldots r$ and $j=r+1, \ldots, n, a_{j i}^{\prime}=0$ where $A^{\prime}=P A P^{T}$. But this then means that $A^{\prime}$ is in the form of (4.1) and hence that $A$ is reducible which is a contradiction. Thus, $D$ must be positive definite as claimed.

Lemma 4.6. Let $A \in \mathbb{R}^{n \times n}$ be Metzler, Hurwitz and irreducible. Suppose that for some non-zero diagonal $D$ in $\mathbb{R}^{n \times n}, A^{T} D+D A=Q \leq 0$. Then $Q$ is also irreducible.

Proof. Once again, we shall argue by contradiction. Suppose that $Q$ is reducible. Then there is some permutation matrix $P$ in $\mathbb{R}^{n \times n}$ such that, if we write $A^{\prime}=P A P^{T}$, $D^{\prime}=P D P^{T}, Q^{\prime}=P Q P^{T}$, then 
(i) $A^{\prime T} D^{\prime}+D^{\prime} A^{\prime}=Q^{\prime} \leq 0$;

(ii) there is some $r$, with $1 \leq r<n$, such that for $i=r+1, \ldots, n, j=1, \ldots, r$, $q_{i j}^{\prime}=0$.

It follows from (ii) that $a_{i j}^{\prime} d_{i}+a_{j i}^{\prime} d_{j}=0$ for $i=r+1, \ldots, n, j=1, \ldots, r$. But from Lemma 4.5, $d_{i}^{\prime}>0$ for $1 \leq i \leq n$, and hence (as $A$ is Metzler) $a_{i j}^{\prime}=0$ for $i=r+1, \ldots, n, j=1, \ldots, r$. This would mean that $A^{\prime}$ was in the form of (4.1) and hence that $A$ was reducible which is a contradiction. Thus $Q$ must be irreducible as claimed.

\section{The rank of matrices in the boundary of $\mathcal{D}_{A}$ :}

The previous technical results establish a number of facts about diagonal matrices on the boundary of $\mathcal{D}_{A}$ where $A$ is an irreducible Metzler, Hurwitz matrix in $\mathbb{R}^{n \times n}$. In particular, we have shown that for any non-zero $D$ on the boundary of $\mathcal{D}_{A}$ :

(i) $D$ must be positive definite;

(ii) $A^{T} D+D A$ is Metzler and irreducible.

Combining (i) and (ii), we have the following result on the boundary structure of the cone $\mathcal{D}_{A}$.

TheOREM 4.7. Let $A \in \mathbb{R}^{n \times n}$ be Metzler, Hurwitz and irreducible. Suppose that $D \in \mathbb{D}^{n \times n}$ satisfies $A^{T} D+D A=Q \leq 0$. Then $\operatorname{rank}(Q)=n-1$, and there is some vector $v \succ 0$ such that $Q v=0$.

Proof. It follows from Lemma 4.4 and Lemma 4.6 that $Q$ is an irreducible Metzler matrix. Furthermore, as $Q \leq 0, \mu(A)=0$. The result now follows from Theorem 4.2.

\section{Conditions for CDLF existence:}

As with the CQLF existence problem, it is possible to use the above results concerning the boundary structure of the cone $\mathcal{D}_{A}$ to derive conditions for CDLF existence for positive LTI systems.

Let $A_{1}, A_{2} \in \mathbb{R}^{n \times n}$ be irreducible Hurwitz, Metzler matrices such that the LTI systems $\Sigma_{A_{1}}, \Sigma_{A_{2}}$ have no CDLF. Further, assume that there exists some non-zero $D_{0} \geq 0$ in $\mathbb{D}^{n \times n}$ such that $A_{i}^{T} D_{0}+D_{0} A_{i}=Q_{i} \leq 0$ for $i=1,2$. Then:

(i) it follows from Theorem 4.7 that the rank of $Q_{i}$ is $n-1$ for $i=1,2$, and that there are vectors $x_{1} \succ 0, x_{2} \succ 0$ with $Q_{i} x_{i}=0$;

(ii) it follows from Theorem 4.3 that there is a unique hyperplane in $\mathbb{D}^{n \times n}$ that separates the open convex cones $\mathcal{D}_{A_{1}}, \mathcal{D}_{A_{2}}$;

(iii) this hyperplane can be parameterized as

$$
\left\{D \in \mathbb{D}^{n \times n}: x_{1}^{T} D A_{1} x_{1}=0\right\},
$$

and

$$
\left\{D \in \mathbb{D}^{n \times n}: x_{2}^{T} D A_{2} x_{2}=0\right\} .
$$

It follows from (iii) and the fact that the cones $\mathcal{D}_{A_{1}}, \mathcal{D}_{A_{2}}$ are disjoint that there is some positive constant $k>0$ such that

$$
x_{1}^{T} D A_{1} x_{1}=-k x_{2}^{T} D A_{2} x_{2}
$$


for all $D \in \mathbb{D}^{n \times n}$. Following similar arguments to those presented in [18], it can be shown that there must be some $D>0$ in $\mathbb{D}^{n \times n}$ with $A_{1}+D A_{2} D$ singular. This fact can then be used to derive the following algebraic necessary and sufficient condition for CDLF existence for pairs of stable positive LTI systems.

TheOREM 4.8. [18] Let $A_{1}, A_{2}$ in $\mathbb{R}^{n \times n}$ be Hurwitz, Metzler and irreducible. Then a necessary and sufficient condition for the stable positive LTI systems, $\Sigma_{A_{1}}$, $\Sigma_{A_{2}}$, to have a CDLF is that $A_{1}+D A_{2} D$ is non-singular for all diagonal $D>0$.

4.2. Linear copositive Lyapunov functions. Given that the trajectories of a positive system are constrained to remain within the non-negative orthant, requiring the existence of a Lyapunov function that works on the entire state space may be an overly restrictive condition for stability. This has led to the consideration of copositive Lyapunov functions for positive systems, and we shall now show how convex cones arise once again in the study of such functions.

We shall only consider the class of linear copositive Lyapunov functions in this paper. Formally, $V(x)=v^{T} x$ is a common linear copositive Lyapunov function for the positive LTI systems $\Sigma_{A_{1}}, \ldots, \Sigma_{A_{m}}$ if $v \in \mathbb{R}^{n}$ is such that $v \succ 0$ and

$$
A_{i}^{T} v \prec 0 \text { for } 1 \leq i \leq m .
$$

In this context, the natural cone to study is given by

$$
\mathcal{V}_{A}=\left\{v \in \mathbb{R}^{n}: v \succ 0 \text { and } A^{T} v \prec 0\right\} .
$$

Theorem 4.1 establishes that for a Metzler matrix $A \in \mathbb{R}^{n \times n}, A$ is Hurwitz if and only if the cone $\mathcal{V}_{A}$ is non-empty. It should be noted that in contrast to the earlier situations, the cone $\mathcal{V}_{A}$ is a polyhedral cone which makes the question of linear copositive function existence less complex than the CQLF or CDLF existence problems. In fact,

$$
\mathcal{V}_{A}=\left\{A^{-T} v: v \prec 0\right\}
$$

\section{Tangent hyperplanes to the cone $\mathcal{V}_{A}$ :}

First of all, in analogy with the approach described above to the CQLF and CDLF existence problems, we shall characterize hyperplanes in $\mathbb{R}^{n}$ that are tangential to the cone $\mathcal{V}_{A}$ at points on its boundary.

Lemma 4.9. Let $A \in \mathbb{R}^{n \times n}$ be Metzler and Hurwitz, and let $z \in \mathbb{R}^{n}$ be such that the hyperplane

$$
\mathcal{H}=\left\{x \in \mathbb{R}^{n}: z^{T} x=0\right\}
$$

is tangential to the cone $\mathcal{V}_{A}$. Then there is some non-zero vector $w \succeq 0$ such that

$$
\mathcal{H}=\left\{x \in \mathbb{R}^{n}:(A w)^{T} x=0\right\} .
$$

Proof. As $\mathcal{H}$ is tangential to the cone $\mathcal{V}_{A}$, it follows that either $z^{T} x<0$ for all $x \in \mathcal{V}_{A}$ or $z^{T} x>0$ for all $x \in \mathcal{V}_{A}$. Without loss of generality, we may assume that 
$z^{T} x<0$ for all $x \in \mathcal{V}_{A}$. It now follows from (4.6) that $z^{T} A^{-T} v<0$ for all $v \prec 0$. Thus, $A^{-1} z=w \succeq 0$, and the result now follows immediately.

\section{Common linear copositive Lyapunov functions:}

The above characterization of hyperplanes that are tangential to the cone $\mathcal{V}_{A}$ can be used to obtain the following result on common linear copositive Lyapunov function existence for a pair of positive LTI systems.

Theorem 4.10. Let $A_{1}, A_{2}$ be Metzler, Hurwitz matrices in $\mathbb{R}^{n \times n}$. Then a necessary and sufficient condition for the systems $\Sigma_{A_{1}}, \Sigma_{A_{2}}$, to have a common linear copositive Lyapunov function is that there are no non-zero vectors $w_{1} \succeq 0, w_{2} \succeq 0$ such that $A_{1} w_{1}+A_{2} w_{2}=0$.

Proof. Necessity: Let $v^{T} x$ be a common linear copositive Lyapunov function for $\Sigma_{A_{1}}, \Sigma_{A_{2}}$. Then $A_{i}^{T} v \prec 0$ for $i=1,2$. Suppose that there are two vectors $w_{1} \succeq 0$, $w_{2} \succeq 0$ such that $A_{1} w_{1}+A_{2} w_{2}=0$. Then

$$
\left(v^{T} A_{1} w_{1}+v^{T} A_{2} w_{2}\right)=0
$$

also. However, the expression (4.7) will be strictly negative unless $w_{1}=0$ and $w_{2}=0$.

Sufficiency: Conversely, assume that the cones $\mathcal{V}_{A_{1}}$ and $\mathcal{V}_{A_{2}}$ do not intersect. Then there is some hyperplane $\mathcal{H}$ through the origin in $\mathbb{R}^{n}$ that separates these cones. This hyperplane will be tangential to both cones at the origin and hence from Lemma 4.9 there are non-zero vectors $w_{1} \succeq 0, w_{2} \succeq 0$ such that

$$
\begin{aligned}
\mathcal{H} & =\left\{x \in \mathbb{R}^{n}: w_{1}^{T} A_{1}^{T} x=0\right\} \\
& =\left\{x \in \mathbb{R}^{n}: w_{2}^{T} A_{2}^{T} x=0\right\} .
\end{aligned}
$$

Moreover, it now follows from the fact that $\mathcal{V}_{A_{1}}$ and $\mathcal{V}_{A_{2}}$ are disjoint that there is some $k>0$ such that

$$
w_{1}^{T} A_{1}^{T}=-k w_{2}^{T} A_{2}^{T} .
$$

The result now follows from (4.8).

5. Non-linear systems, Lyapunov functions and convex cones. In the previous sections, we have studied several convex cones that arise when considering the stability question for dynamical systems constructed by switching between a family of stable linear time-invariant systems. We shall next consider ways of extending some of these ideas to non-linear systems, highlight the role played by convex cones in this context and present some initial results on the CQLF existence problem for non-linear systems.

\section{The cone $\mathcal{P}_{f}$ :}

Let $f: \mathbb{R}^{n} \rightarrow \mathbb{R}^{n}$ be a non-linear vector field and denote the corresponding dynamical system by $\Sigma_{f}$,

$$
\Sigma_{f}: \dot{x}=f(x)
$$


In analogy with the cone $\mathcal{P}_{A}$ for LTI systems, we introduce the set $\mathcal{P}_{f}$ defined by

$$
\mathcal{P}_{f}=\left\{P=P^{T}>0: x^{T} P f(x)<0 \text { for all non-zero } x \in \mathbb{R}^{n}\right\} .
$$

If $P \in \mathcal{P}_{f}$, then $V(x)=x^{T} P x$ is said to be a quadratic Lyapunov function for the non-linear system $\Sigma_{f}$. It is straightforward to check that $\mathcal{P}_{f}$ is a convex cone.

Given two vector fields $f_{1}, f_{2}$, we say that the corresponding systems, $\Sigma_{f_{1}}, \Sigma_{f_{2}}$ have a common quadratic Lyapunov function (CQLF) if the cones $\mathcal{P}_{f_{1}}, \mathcal{P}_{f_{2}}$ have a non-empty intersection. As we are interested in the question of CQLF existence, throughout this section we shall assume that the systems considered have quadratic Lyapunov functions. However, it should be noted that while the stability of the LTI system $\Sigma_{A}$ is equivalent to the cone $\mathcal{P}_{A}$ being non-empty, this need not in general be the case for non-linear systems.

\section{The relationship between $\mathcal{P}_{f}$ and $\mathcal{P}_{f^{-1}}$ :}

Theorem 3.1 establishes that for a stable LTI system $\Sigma_{A}$, the cones $\mathcal{P}_{A}$ and $\mathcal{P}_{A^{-1}}$ coincide exactly. In the next result, we show that for an invertible vector field $f$, this result also holds for non-linear systems. ${ }^{2}$

LEMMA 5.1. Let $f: \mathbb{R}^{n} \rightarrow \mathbb{R}^{n}$ be an invertible vector field, with inverse $f^{-1}$. Then for any positive definite matrix $P=P^{T}>0$,

$$
x^{T} P f(x)<0 \text { for all non-zero } x \in \mathbb{R}^{n}
$$

if and only if

$$
x^{T} P f^{-1}(x)<0 \text { for all non-zero } x \in \mathbb{R}^{n} \text {. }
$$

Proof. Let $P$ be a positive definite matrix in $\mathbb{R}^{n}$ such that

$$
x^{T} \operatorname{Pf}(x)<0 \text { for all non-zero } x \in \mathbb{R}^{n} .
$$

Now let $y$ be any non-zero vector in $\mathbb{R}^{n}$. Then, $f^{-1}(y)$ will also be non-zero and it follows from (5.3) that $\left(f^{-1}(y)\right)^{T} P y<0$, and thus that $y^{T} P f^{-1}(y)<0$. Hence, we have shown that

$$
x^{T} \operatorname{Pf}(x)<0 \quad \text { for all non-zero } x \in \mathbb{R}^{n}
$$

implies that

$$
x^{T} P f^{-1}(x)<0 \text { for all non-zero } x \in \mathbb{R}^{n} .
$$

The converse follows by interchanging the roles of $f$ and $f^{-1}$ in the previous argument.

\footnotetext{
${ }^{2}$ The second named author wishes to express his gratitude to Dr. Paul Curran of University College Dublin for helpful discussions on the subject of this result.
} 


\section{Necessary conditions for CQLF existence:}

It is known [27, 31] that if two stable LTI systems $\Sigma_{A_{1}}, \Sigma_{A_{2}}$ have a CQLF then the matrix pencils $A_{1}+\gamma A_{2}$ and $A_{1}+\gamma A_{2}^{-1}$ are non-singular for all $\gamma>0$. Equivalently, the matrix products $A_{1} A_{2}^{-1}, A_{1} A_{2}$ have no negative real eigenvalues. We now derive corresponding necessary conditions for CQLF existence for a pair of non-linear systems $\Sigma_{f_{1}}, \Sigma_{f_{2}}$.

Lemma 5.2. Let $f_{1}: \mathbb{R}^{n} \rightarrow \mathbb{R}^{n}, f_{2}: \mathbb{R}^{n} \rightarrow \mathbb{R}^{n}$ be invertible vector fields, and suppose that there exists a CQLF for the associated systems $\Sigma_{f_{1}}, \Sigma_{f_{2}}$. Then for all $\gamma>0$, and all non-zero $x \in \mathbb{R}^{n}$ :

$$
\begin{array}{r}
f_{1}(x)+\gamma f_{2}(x) \neq 0 \\
f_{1}^{-1}(x)+\gamma f_{2}(x) \neq 0
\end{array}
$$

Proof. Let $V(x)=x^{T} P x$ be a CQLF for $\Sigma_{f_{1}}, \Sigma_{f_{2}}$. Then it follows using Lemma 5.1 that for all non-zero $x \in \mathbb{R}^{n}$,

$$
x^{T} P f_{1}(x)<0, x^{T} P f_{2}(x)<0, x^{T} P f_{1}^{-1}(x)<0 .
$$

Thus, for any non-zero $x$ in $\mathbb{R}^{n}$ and any $\gamma>0$,

$$
\begin{aligned}
x^{T} P\left(f_{1}(x)+\gamma f_{2}(x)\right) & <0 \\
x^{T} P\left(f_{1}^{-1}(x)+\gamma f_{2}(x)\right) & <0 .
\end{aligned}
$$

The result now follows immediately.

REMARK 5.3. The above result shows that if there exists a CQLF for the systems $\Sigma_{f_{1}}, \Sigma_{f_{2}}$, then there can be no non-zero vector $x$ in $\mathbb{R}^{n}$ for which $f_{1}^{-1}\left(f_{2}(x)\right)=-\lambda x$, or $f_{1}\left(f_{2}(x)\right)=-\lambda x$ with $\lambda>0$. Thus Lemma 5.2 extends the fact that a necessary condition for two stable LTI systems, $\Sigma_{A_{1}}, \Sigma_{A_{2}}$, to have a CQLF is that the matrix products $A_{1}^{-1} A_{2}$ and $A_{1} A_{2}$ have no negative real eigenvalues.

\section{Tangent hyperplanes and CQLF existence for non-linear systems:}

Finally for this section, we show how the arguments used earlier to derive Theorem 3.3 can be carried over to a non-linear setting. In the statement of the next theorem, we assume that the vector fields being considered are homogeneous of degree one, where a mapping $f: \mathbb{R}^{n} \rightarrow \mathbb{R}^{n}$ is said to be homogeneous of degree one if $f(\lambda x)=$ $\lambda f(x)$ for all $\lambda \in \mathbb{R}, x \in \mathbb{R}^{n}$.

THEOREM 5.4. Let $f: \mathbb{R}^{n} \rightarrow \mathbb{R}^{n}$ be invertible and homogeneous of degree one. Suppose that $P_{0}$ lies on the boundary of $\mathcal{P}_{f}$, and that there is some non-zero vector $x_{0} \in \mathbb{R}^{n}$ such that

$$
\left\{x \in \mathbb{R}^{n}: x^{T} \operatorname{Pf}(x)=0\right\}=\left\{x \in \mathbb{R}^{n}: x=\lambda x_{0} \text { for } \lambda \in \mathbb{R}\right\}
$$

Then:

(i) there is a unique hyperplane tangential to the cone $\mathcal{P}_{f}$ at $P_{0}$; 
(ii) this plane is given by

$$
\left\{H \in \mathbb{S}^{n \times n}: x_{0}^{T} H f\left(x_{0}\right)=0\right\} .
$$

Proof. This result can be proven in the same way as Theorem 3.2 using the homogeneity of the vector field $f$.

In analogy with the linear case, the above characterization of hyperplanes tangential to the cone $\mathcal{P}_{f}$ can be used to obtain the following non-linear version of Theorem 3.3 .

TheOREM 5.5. Let $f_{1}: \mathbb{R}^{n} \rightarrow \mathbb{R}^{n}, f_{2}: \mathbb{R}^{n} \rightarrow \mathbb{R}^{n}$ be invertible vector fields, homogeneous of degree one, such that the systems $\Sigma_{f_{1}}, \Sigma_{f_{2}}$ have no CQLF. Furthermore, suppose that there is some positive definite $P=P^{T}>0$ such that, for $i=1,2$ :

(i) $x^{T} P f_{i}(x) \leq 0$ for all $x$ in $\mathbb{R}^{n}$;

(ii) there is a non-zero vector $x_{i} \in \mathbb{R}^{n}$ such that

$$
\left\{x \in \mathbb{R}^{n}: x^{T} P f_{i}(x)=0\right\}=\left\{x \in \mathbb{R}^{n}: x=\lambda x_{i} \text { for } \lambda \in \mathbb{R}\right\} .
$$

Then either

(i) there exists some non-zero $x$ in $\mathbb{R}^{n}$ and some $\gamma>0$ such that $f_{1}(x)+\gamma f_{2}(x)=0$ or

(ii) there exists some non-zero $x$ in $\mathbb{R}^{n}$ and some $\gamma>0$ such that $f_{1}^{-1}(x)+$ $\gamma f_{2}(x)=0$.

Note that under the hypotheses of Theorem 5.5, the necessary conditions for CQLF existence given in Lemma 5.2 are violated.

6. Concluding remarks. In this paper, we have considered three types of Lyapunov function that are closely related to the theory of convex cones. Specifically, we have presented results on the structure of cones that arise in the study of quadratic Lyapunov functions for both linear and non-linear systems, as well as a number of results on cones of diagonal Lyapunov functions and linear copositive Lyapunov functions for positive linear systems. The relevance of the structure of these cones to the problem of common Lyapunov function existence has been discussed, and it has been demonstrated how this structure can be used to derive conditions for the existence of such functions.

Acknowledgments. This work was partially supported by Science Foundation Ireland grant 00/PI.1/C067 and Science Foundation Ireland Basic Research Grant 04/BR/m0061 and Science Foundation Ireland Grant 04/INI/1478. Science Foundation Ireland is not responsible for any use of data appearing in this publication.

\section{REFERENCES}

[1] T. Ando. Sets of matrices with a common Lyapunov solution. Archiv der Mathematik, 77:76-84, 2001.

[2] A. Berman and R.J. Plemmons. Non-negative matrices in the mathematical sciences. SIAM Classics in Applied Mathematics, Philadelphia, 1994.

[3] S. Boyd, L. El Ghaoui, E. Feron, and V. Balakrishnan. Linear Matrix Inequalities in System and Control Theory. SIAM, Philadelphia, 1994. 
[4] N. Cohen and I. Lewkowicz. A necessary and sufficient criterion for the stability of a convex set of matrices. IEEE Transactions on Automatic Control, 38(4):611-615, 1993.

[5] N. Cohen and I. Lewkowicz. Convex invertible cones and the Lyapunov equation. Linear Algebra and its Applications, 250(1):105-131, 1997.

[6] N. Cohen and I. Lewkowicz. A pair of matrices sharing common Lyapunov solutions - a closer look. Linear Algebra and its Applications, 360:83-104, 2003.

[7] R. DeCarlo, M. Branicky, S. Pettersson, and B. Lennartson. Perspectives and results on the stability and stabilisability of hybrid systems. Proceedings of the IEEE, 88(7):1069-1082, 2000.

[8] L. Farina and S. Rinaldi. Positive linear systems. Wiley Interscience Series, New York, 2000.

[9] D. Hershkowitz. On cones and stability. Linear algebra and its applications, 275/276:249-259, 1998.

[10] R. Horn and C. Johnson. Matrix Analysis. Cambridge University Press, Cambridge, 1985.

[11] R. Horn and C. Johnson. Topics in Matrix Analysis. Cambridge University Press, Cambridge, 1991.

[12] A. Jadbabaie, J. Lin, and A. S. Morse. Co-ordination of groups of mobile autonomous agents using nearest neighbour rules. IEEE Transactions on Automatic Control, 48(6):988-1001, 2003.

[13] C. King and R. Shorten. A spectral condition for the existence of a CQLF. In American Control Conference, 2004.

[14] I. Lewkowicz. A necessary condition for quantitative exponential stability of state spacesystems. Systems \& Control Letters, 38:1-4, 1999.

[15] Daniel Liberzon and A. Stephen Morse. Basic problems in stability and design of switched systems. IEEE Control Systems Magazine, 19(5):59-70, 1999.

[16] R. Loewy. On ranges of real Lyapunov transformations. Linear Algebra and its Applications, 13:79-89, 1976.

[17] David G. Luenberger. Introduction to Dynamic Systems. John Wiley \& Sons, New York, 1979.

[18] O. Mason and R. Shorten. On the simultaneous diagonal stability of a pair of positive linear systems. To appear in Linear Algebra and its Applications, 2004.

[19] N.H. Mc Clamroch and I. Kolmanovsky. Performance benefits of hybrid control design for linear and nonlinear systems. Proceedings of the IEEE, 88(7):1083-1096.

[20] K.R. Meyer. On the existence of Lyapunov functions for the problem of Lur'e. SIAM Journal on Control and Optimization, 3(3):373-383, 1966.

[21] Y. Mori, T. Mori, and Y. Kuroe. A solution to the common Lyapunov function problem for continuous time systems. In proceedings of 36th Conference on Decision and Control, San Diego, 1997.

[22] K. S. Narendra and J. Balakrishnan. A common Lyapunov function for stable LTI systems with commuting $\mathcal{A}$-matrices. IEEE Transactions on Automatic Control, 39(12):2469-2471, 1994.

[23] K. S. Narendra and J. Balakrishnan. Adaptive control using multiple models. IEEE Transactions on Automatic Control, 42(3):171-187, 1997.

[24] K. S. Narendra and R. M. Goldwyn. A geometrical criterion for the stability of certain nonlinear non-autonomous systems. IEEE Transactions on Circuit Theory, 11(3):406-407, 1964.

[25] R. T. Rockafellar. Convex Analysis. Princeton University Press, Princeton, 1970.

[26] R. Shorten, D. Leith, J. Foy, and R. Kilduff. Towards an analysis and design framework for congestion control in communication networks. In Proceedings of the 12th Yale workshop on adaptive and learning systems, 2003.

[27] R. N. Shorten, O. Mason, F. O' Cairbre, and P. Curran. A unifying framework for the SISO Circle Criterion and other quadratic stability criteria. International Journal of Control, $77(1): 1-8,2004$.

[28] R. N. Shorten and K. S. Narendra. On the stability and existence of common Lyapunov functions for linear stable switching systems. In proceedings 37th Conference on Decision and Control, Tampa, Florida, 1998. 
[29] R. N. Shorten and K. S. Narendra. On common quadratic Lyapunov functions for pairs of stable LTI systems whose system matrices are in companion form. IEEE Transactions on Automatic Control, 48(4):618-621, 2003.

[30] R. N. Shorten, K. S. Narendra, and O. Mason. A result on common quadratic Lyapunov functions. IEEE Transactions on Automatic Control, 48(1):110-113, 2003.

[31] Robert N. Shorten and Kumpati S. Narendra. Necessary and sufficient conditions for the existence of a common quadratic Lyapunov function for a finite number of stable second order linear time-invariant systems. International Journal of Adaptive Control and Signal Processing, 16:709-728, 2003. 